\title{
Philosophiques
}

\section{Diane Lamoureux, Les possibles du féminisme. Agir sans \\ " nous ", Les Éditions du Remue-ménage, Montréal, 2016}

\section{Margaux Ruellan}

Volume 43, numéro 2, automne 2016

URI : https://id.erudit.org/iderudit/1038225ar

DOI : https://doi.org/10.7202/1038225ar

Aller au sommaire du numéro

Éditeur(s)

Société de philosophie du Québec

ISSN

0316-2923 (imprimé)

1492-1391 (numérique)

Découvrir la revue

Citer ce compte rendu

Ruellan, M. (2016). Compte rendu de [Diane Lamoureux, Les possibles $d u$

féminisme. Agir sans «nous », Les Éditions du Remue-ménage, Montréal, 2016].

Philosophiques, 43(2), 544-549. https://doi.org/10.7202/1038225ar

Ce document est protégé par la loi sur le droit d'auteur. L'utilisation des services d'Érudit (y compris la reproduction) est assujettie à sa politique d'utilisation que vous pouvez consulter en ligne.

https://apropos.erudit.org/fr/usagers/politique-dutilisation/ 
science, la forme particulière que doit prendre cet examen varie d'un auteur à l'autre. Comment la critique des modèles naturalistes doit-elle s'opérer? Andler accepte-t-il le pluralisme méthodologique de Paul Feyerabend ${ }^{14}$, les exigences de diversité et d'impartialité d'Helen Longino ${ }^{15}$ ou les exigences délibératives encadrant les questions et méthodes scientifiques? On a l'impression que l'argument d'Andler est compatible avec n'importe quelle interprétation du rôle de la critique dans les sciences. Dans le contexte où personne ne semble rejeter le rôle de la critique pour l'avancement des sciences et de la philosophie, Andler gagnerait à exposer clairement qui sont ses interlocuteurs.

Ces interrogations en ce qui touche à la problématique et à la notion de critique ne remettent toutefois pas en question l'œuvre prise dans son ensemble. Andler expose des sujets complexes de manière abordable et structurée, et les questions qui sont posées dans ce livre sont déterminantes pour l'avenir des sciences et de la philosophie. Il s'agit d'une contribution pertinente sur la question du naturalisme.

MARC-KEVIN DAOUST

Université de Montréal marc-kevin.daoust@umontreal.ca

\section{Diane Lamoureux, Les possibles du féminisme. Agir sans «nous», Les Éditions du Remue-ménage, Montréal, 2016.}

À l'heure où le féminisme subit les assauts du néo-conservatisme et du néo-libéralisme, autant au Québec qu'ailleurs, la publication de cet ouvrage est une bouffée d'air pour celles et ceux qui croient au développement voire au renouvellement d'une pensée féministe critique, inclusive et solidaire. Les Éditions du Remue-ménage rassemblent ici des textes publiés par Diane Lamoureux dans des cadres divers qui tissent habilement les liens complexes entre théories philosophiques, transformations politiques et sociales, et pratiques militantes au Québec. Professeure de philosophie et de sociologie politique à l'Université Laval, Diane Lamoureux n'a jamais versé dans l'élitisme universitaire. Au contraire, elle défend une pensée féministe radicale, solidaire des exclus et exclues de tous horizons, et qui puise dans la pratique militante à la fois le courage et la validation théorique.

Les articles s'articulent autour de deux tensions qui sont au fondement des débats féministes depuis leurs origines. Le premier "paradoxe (p. I06) » concerne le contenu des revendications féministes. Les féministes cherchent

14. Paul Feyerabend. Contre la méthode. Esquisse d'une théorie anarchiste de la connaissance, Paris, Éditions du Seuil, I979.

15. Helen E. Longino. Science as Social Knowledge: Values and Objectivity in Scientific Inquiry, Princeton, Princeton University Press, I990. 
à susciter une prise de conscience, d'abord chez les femmes, qu'il existe bel et bien une nature commune aux sexismes subis par chacune dans sa situation particulière. Au bout du compte, le but de cet éclairage est la libération des femmes comme personnes différenciées et leur autonomie en tant que citoyennes. Cela suppose qu'elles aient les capacités de s'extraire d'une catégorisation et de toute assignation identitaire; or le fait de souligner la communauté des oppressions dont les femmes font l'expérience, n'est-ce pas là renforcer une catégorisation sociale dont elles souhaitent justement se départir? Comment comprendre et agir contre les injustices qui s'adressent systématiquement à un groupe social sans tomber dans le piège d'homogénéiser les expériences vécues?

La deuxième tension découle directement de ce qui précède et concerne la constitution d'un mouvement social pluraliste. Plus sa réalisation devenait possible - grâce aux acquis juridiques, sociaux et politiques du mouvement féministe québécois des années I960 aux années I980 - plus le processus d'individuation des femmes se traduisait par des différends au sein des groupes de «femmes» (p. I 53) et rendait difficile la capacité des femmes à «faire mouvement». Il fallait démontrer que la pluralité et le dissensus sont des signes d'émancipation politique des femmes à travers leurs différences singulières, et couper définitivement l'herbe sous le pied des détracteurs qui profitaient de la situation pour opposer les «féministes" aux «femmes». Comment alors définir une fin commune sans brimer les voies dissidentes? Comment inclure la diversité et les divergences d'opinions sans nuire à la représentation et à la réalisation efficace du projet féministe? Finalement, comment «agir sans [un] «nous» (p. I47)»?

Chaque article constitue une manière différente de répondre à ces enjeux. La position de Lamoureux est aussi originale que radicale. Critique de l'idée d'une "troisième vague féministe" (p. I9I), inspirée par Hannah Arendt, Simone de Beauvoir et Françoise Collin, mais aussi par la théorie critique de Michel Foucault, Judith Butler, Jacques Rancière et Iris Marion Young, elle défend la subversion de toute tentative d'identification des femmes à un "Autre » subalterne, ayant comme point de mire l'acquisition du statut de sujet politique pour toutes. Les moyens n'étant pas dissociables des fins, le défi de cette lutte consiste justement à déterminer une méthode militante prenant acte de l'intersection des oppressions et de la singularité des expériences vécues, qui permettrait de former des coalitions solidaires respectueuses de l'autonomie de chaque partie.

L'ouvrage s'ouvre avec un article intitulé "Comment l'égalité entre les femmes et les hommes est devenue une "valeur fondamentale de la société québécoise" " qui fait le point sur les nombreuses transformations de la législation québécoise depuis les années I960. L'acquisition du droit de vote en I940 ne suffisant pas à consacrer l'égalité des femmes comme sujet politique dans les faits, les militantes se sont penchées sur des enjeux 
comme la famille, la sexualité, le travail et la violence qui empêchaient les femmes de s'émanciper politiquement. Leurs combats ont permis, entre autres, la reconnaissance de l'égalité de l'autorité parentale par la loi 89 en I980; la levée de l'interdiction de l'avortement en I988 et la généralisation de l'accès à la contraception; l'imposition de l'équité salariale, la mixité dans les écoles et l'indifférenciation des programmes solaires; ou encore la reconnaissance juridique du viol conjugal et du harcèlement sexuel au travail et à l'université.

Si les articles qui suivent rappellent ces acquis du mouvement féministe, c'est pour mieux souligner leur précarité, dans un contexte de néolibéralisation des politiques publiques et de résurgence du masculinisme néo-conservateur. L'égalité entre les hommes et les femmes - maintenant déclarée "valeur fondamentale de la société québécoise» — serait-elle acquise et le féminisme dépassé ? Vivrions-nous maintenant dans un "monde postféministe» (p. I73) ? Bien au contraire, les inégalités entre les sexes persistent, comme en témoignent la division genrée du travail - qui confie systématiquement aux femmes les soins et l'assistance (de la garde d'enfants à la prostitution) ainsi que les emplois les moins bien rémunérés. Par ailleurs, il est encore et toujours plus difficile pour les femmes de concilier vie de famille, études et travail salarié du fait du désengagement général de l'État dans les services publics (Centres de la Petite Enfance, accès aux soins de santé), les programmes d'aides aux familles immigrantes et les droits des travailleurs. Dans «Service ou politique: quelques dilemmes du mouvement des femmes» (p. 55), Lamoureux démontre que l'intégration subalterne à l'appareil d'État des services prodigués par les diverses « collectives $^{1}$ » féministes - comme l'hébergement des femmes violentées, par exemple - se solde à la fois par un plus grand contrôle de ces services du fait de leur dépendance financière envers l'État (p. 66-67), et par la déresponsabilisation des instances publiques vis-à-vis de la justice sociale (p. 69). Dans le même ordre d'idée, Lamoureux se fait critique aussi bien de l'économie sociale (p. 75-76), que de l'allocation universelle (p. I I I) et même des programmes de micro-crédit (p. 234-235) qui renforcent la dépendance des femmes à un encadrement financier principalement masculin et blanc, font reposer le fardeau de la pauvreté sur les épaules des "citoyens responsables» et autorisent l'État à se retirer progressivement de l'assistance aux groupes sociaux les plus discriminés et les plus démunis.

1. Diane Lamoureux emploie volontairement le féminin pluriel pour parler des collectifs militants féministes - une manière de souligner que leurs membres sont essentiellement des femmes et que leurs luttes et leurs méthodes s'inscrivent dans un combat féministe. Afin de restituer honnètement la pensée de l'auteure, j'ai donc préféré laisser ce terme dans sa forme du texte original. 
Les articles «Objectiver les personnes, réifier les situations» (p. 99), "Féminins singuliers et féminins pluriels (p. I2I) et "Agir sans "nous" " (p. I47) constituent sans doute le cœur de la théorie féministe de la liberté où Diane Lamoureux s'attaque plus directement aux deux tensions précédemment évoquées. La libération commence par le dévoilement de l'oppression subie par le groupe des "femmes", catégorie sociale qui permet l'assignation à une identité subalterne et qu'il s'agit alors de se réapproprier pour mieux s'en départir (p. I06). La catégorisation est un outil de domination (Foucault, p. I05) puisqu'elle permet la réification des personnes (Nussbaum, voir p. IOI-IO5) et leur pleine exploitation par le capitalisme et le patriarcat. Le fait que les femmes soient constamment ramenées à une identité féminine dévalorisante est une manière de nier leur subjectivité et de miner les bases d'une relation de réciprocité nécessaire à l'affirmation de soi comme sujet politique (p. I28). Qu'entend Lamoureux par le concept de «subjectivation politique ", emprunté à Rancière? La critique féministe du sujet moderne par Mary Wollstonecraft, Carol Pateman et Iris Marion Young (p. I 25-I 26) sert de point de départ pour une réhabilitation non essentialiste du sujet qui échappe au dualisme de l'assimilation et de la différentiation vis-à-vis d'un sujet paré des attributs de la masculinité. Il s'agit de penser le sujet politique en rupture radicale avec toute forme d'identification et de caractérisation ontologique ou métaphysique (p. I60-I6I), afin "d'envisager une communauté politique diversifiée dont le ciment ne soit pas l'identité mais le lien politique (p. I64)». La subjectivation politique se réalise donc par la prise de parole et l'action qui se tiennent dans un contexte relationnel toujours particulier (p. I45). Lamoureux prend ici ses distances avec le féminisme post-moderne de Butler qui annonçait la mort du sujet (p. I68), tout en gardant l'idée d'une approche différencialiste de la subjectivation. La notion de sujet politique ainsi réévaluée se réalise par l'individuation de chacune à travers ses différences et amène à redéfinir la citoyenneté à l'aune d'un impératif d'inclusion de toutes les singularités.

On le voit, ce n'est pas tant l'égalité que la pratique d'une liberté au quotidien - conçue dans les terme de l'autonomie personnelle - qui constitue l'horizon de la lutte féministe. Lamoureux se fait même critique de l'égalitarisme strict qui tend vainement à homogénéiser les situations (p. 5I) et défavorise ceux qui sont moins armés pour faire valoir leurs droits (p. 92). Certes, le traitement égalitaire des femmes suppose qu'elles soient considérées comme des sujets dignes de droits - et non comme des êtres de besoins, des victimes (p. 70-7I), ou pire encore, comme un groupe d'intérêts (p. 96) - et, en cela, constitue un premier pas vers la capacité à s'individualiser. Toutefois, certaines transformations sociales et politiques, qui ne passent pas uniquement par la judiciarisation, sont nécessaires pour que les femmes jouissent d'une réelle autonomie personnelle. Celle-ci demande une indépendance individuelle, certainement garantie par le droit, mais aussi par l'acquisition d'un revenu suffisant ainsi que des libertés sexuelles et reproductives 
(p. 47-48). L'autonomie personnelle s'accompagne d'une autonomie sur le plan politique et sur le plan organisationnel (p. 46-47), c'est-à-dire la reconnaissance de l'indépendance des mouvements féministes dans la définition de leurs enjeux de lutte et leurs choix d'organisation (comme par exemple la non-mixité) et du sens en soi du combat contre le patriarcat qui ne saurait être rabattu sous des enjeux considérés comme plus importants (lutte anticapitaliste, par exemple).

Cependant, il reste difficile de savoir quel rôle doivent jouer les institutions juridiques et l'État dans l'accession des femmes au statut de sujet politique et à l'autonomie. Il y a sûrement l'idée sous-entendue d'une interrelation complexe entre des protections juridiques, la représentation politique adéquate des revendications plurielles, l'engagement sérieux de l'État dans la lutte contre le sexisme structurel et la considération pour l'autonomie des groupes, associations et organismes communautaires de la société civile qui prodiguent des services d'accompagnement locaux personnalisés. On comprend au fil de la lecture, sans que cela soit dit explicitement, que l'autonomie personnelle et la subjectivation politique des femmes requièrent l'engagement conjoint des pôles judiciaire, politique et social ainsi que l'action combinée de mouvements solidaires à différentes échelles.

Enfin, l'ouvrage ne cesse de démontrer que la défense féministe des libertés constitue la base d'un discours général sur la justice sociale et la démocratie (p. I40, I 89, 205 et 26I). L'enjeu des luttes féministes - il convient d'en parler au "féminin pluriel » — serait donc bien «le monde» au sens arendtien du terme, c'est-à-dire l'espace partagé par une communauté politique. En effet, le féminisme ne s'intéresse pas qu'aux intérêts des femmes, mais, conscient de l'intersectionnalité des multiples mécanismes de domination sexistes, racistes et capitalistes (p. 253 et 257), il cherche à concevoir des liens de solidarité avec d'autres collectifs. Diane Lamoureux façonne ainsi un concept de solidarité politique qui puisse permettre l'inclusion de différentes parties dans un projet commun soucieux de la préservation de l'autonomie de chacune. Cette solidarité politique est d'abord éprouvée à l'intérieur même du mouvement féministe. Les "collectives" féministes, en prenant acte de leur pluralité (p. I38), ont développé des rapports critiques et solidaires, faisant de leurs différences et de leurs différends des ressources plutôt que des obstacles à l'individuation. Mais en quoi consiste cette solidarité ? Lamoureux part du concept de "sororité ", pendant féminin de la fraternité, qui signifie le soutien réciproque entre femmes fondés sur le partage d'une identité féminine commune, pour envisager son dépassement par l'idée de solidarité politique, qui est le "résultat d'une délibération politique reposant sur une prise en compte des différences (p. I07) ». Aussi la solidarité interne aux "collectives » féministes pourrait-elle servir de modèle pour concevoir les fondements d'un projet commun de justice et de démocratie dans notre société québécoise. 
De la même manière, le mouvement féministe représente une source d'inspiration pour d'autres luttes, et notamment celle du socialisme contre l'exploitation capitaliste. L'article «Et si le socialisme avait à apprendre du féminisme?» (p. 215) souligne au moins trois leçons que les groupes militants pour la justice sociale devraient retenir du militantisme féministe. Premièrement, les féministes ont insisté sur l'adéquation entre les moyens et les fins (p. 216-219): si l'on cherchait à combattre les formes diverses de l'oppression sociale, il fallait que les pratiques militantes veillent à ne pas les reconduire à l'interne. Les mouvements se sont donc organisés en réseaux horizontaux et décentralisés afin de mieux intégrer la diversité et de fédérer les efforts de chaque collectif sans les confondre. Deuxièmement, le féminisme a redonné ses lettres de noblesse à la pratique, en insistant sur le vécu des dominés et dominées comme ressource cognitive et sur la concrétisation de l'action politique (p. 2I9-22I). Enfin, il cherche à revaloriser l'action directe et la subversion quotidienne des rapports de domination dans les contextes sociaux du présent, plutôt que d'attendre un hypothétique moment opportun ou révolutionnaire pour transformer le monde.

La pensée féministe de Diane Lamoureux prend à bras le corps la complexité d'une lutte sociale et politique qu'elle souhaite radicale et vive. Si chacun des articles a la qualité de restituer l'entièreté d'une réflexion sinueuse, le lecteur reste toutefois un peu sur sa faim en ce qui concerne l'élaboration théorique et surtout la réalisation pratique de certaines notions dont Diane Lamoureux nous a pourtant persuadées de la pertinence: celle de "solidarité critique» et celle de "radicalité", par exemple, sont des intuitions qui demanderaient à être développées. Enfin, on se demande comment devrait fonctionner en pratique un "nous» collectif intégrant la différence et le différend. Mais ce flou témoigne sûrement encore une fois du souci qu'a l'auteure de laisser aux «collectives » leur pleine autonomie pour interpréter et réaliser en pratique ces exigences théoriques.

MARGAUX RUELLAN

Université de Montréal 